\title{
Development and physico-mechanical characterization of carrageenan and poloxamer based lyophilized matrix as a potential buccal drug delivery system
}

\author{
Farnoosh Kianfar, Isaac Ayensu, Joshua S. Boateng*
}

Department of Pharmaceutical, Chemical \& Environmental Sciences, School of Science, University of Greenwich, Medway Campus, Central Avenue, Chatham Maritime, Kent ME4 4TB, UK

Abbreviations: $\kappa$-Carrageenan 911 (CAR); Differential scanning calorimetry (DSC); Hot stage microscopy (HSM); Polyethylene glycol (PEG); Poloxamer (POL); Glass transition $\left(\mathrm{T}_{\mathrm{g}}\right)$; thermogravimetric analysis (TGA); Scanning electron microscopy (SEM); X-ray powder diffraction (XRPD)

Keywords: Adhesion, Carrageenan, Glass transition, Swelling capacity, Wafer

*Corresponding author: Department of Pharmaceutical, Chemical and Environmental Sciences, School of Science, University of Greenwich at Medway, Central Avenue, Chatham Maritime, Kent, UK ME4 4TB. Tel: + 44 (0) 208331 8980; Fax: +44 (0) 208331 9805,

E-mail address: J.S.Boateng@gre.ac.uk (Dr Joshua Boateng). 


\begin{abstract}
The objective of this study was the formulation development of polymeric mucoadhesive lyophilized wafers as a matrix for potential buccal drug delivery. Differential scanning calorimetry (DSC) was used to develop an optimum freeze-cycle, incorporating an annealing step. The wafers were prepared by lyophilization of gels containing three polymers, $\kappa-$ carrageenan (CAR 911), poloxamer (P407) and polyethylene glycol 600 (PEG 600). The formulations were characterised using texture analysis (for mechanical and mucoadhesion properties), hydration studies, thermogravimetric analysis (TGA), DSC, X-ray powder diffraction (XRPD) and scanning electron microscopy (SEM). DSC showed the eutectic temperature $\left(12.8^{\circ} \mathrm{C}\right)$ of the system where the liquid solution and pure solids both existed at a fixed pressure which helped determine the freeze-annealing cycle at $-55^{\circ} \mathrm{C}$ for seven hours. Mechanical resistance to compression, hydration and mucoadhesion studies showed that optimized wafers were obtained from aqueous gels containing $2 \%$ w/w CAR 911, $4 \%$ w/w P407 and 4.4\% w/w PEG 600. TGA showed residual water of approximately $1 \%$ and SEM showed a porous polymeric network that made ease of hydration possible.
\end{abstract}




\section{Introduction}

Current research in many industries (e.g. food and pharmaceutical) has been directed towards a new generation of polymeric matrices for systemic drug delivery or topical application. Lyophilisation (freeze-drying) has been used in food formulations for a long time $^{1}$ for example lyophilization of soup, coffee and strawberries, avoiding the cold chain supply and products can retain their original volume and shape. Pharmaceutical and biotechnology industries subsequently began employing the technique to improve the stability of protein (enzymes, genes, sera and vaccines) based formulations ${ }^{2-5}$. The fundamental physico-chemical processes occurring during lyophilization are known to determine and affect achievement of stable and well-designed lyophilized pharmaceutical products ${ }^{5}$. Though the cost of the specialized equipment employed for lyophilisation can be considerably high, it is particularly useful for formulation and storage of thermo-labile products. In addition, lyophilized products do not necessarily need to be refrigerated and can be stored at ambient temperatures ${ }^{6}$.

The first stage of lyophilization comprises a freezing step where ice crystals form and the original solute becomes highly concentrated. As the temperature falls below the glass transition temperature of the concentrated frozen solute, the matrix is changed into a viscous metastable glass. Incomplete crystallization may lead to sample collapse or formation of mixtures of different polymorphic forms that causes problems in reproducible manufacturing and characterization ${ }^{7}$. To overcome this problem, and achieve complete crystallization, thermal treatment or "annealing", is required. Annealing involves warming the sample above the $\mathrm{T}_{\mathrm{g}}$ (determined through DSC analysis) and maintaining for a given time period (up to several hours). This helps to reduce the viscosity and increase the mobility of the solute molecules, which prevents premature solute crystallization. However, the final elevated temperature should remain below the eutectic or collapse point to avoid ice melting. 
Temperatures above this point results in the metastable glass becoming fluidised and consequently causes movements of the polymer molecules which orient themselves into a crystalline lattice to produce a binary eutectic with ice ${ }^{8}$. The annealing procedure must be integrated into the freezing step for all formulations containing one or more crystallisable compounds. This increases both solute and ice crystal formation, which reduces the product resistance to moisture transfer, faster water vapour transport and shorter primary drying times $^{8,9}$.

Wafers are prepared by lyophilization of aqueous polymer gels to form a porous polymeric inter-connecting network ${ }^{10}$. To obtain an efficient mucosal delivery system, swelling and mucoadhesion characteristics must be optimized as they affect functional characteristics such as residence time and drug release profiles ${ }^{11}$. An initial swelling step is required for the formation of adhesive forces at the mucosal surface which ultimately results in bio (muco)-adhesion ${ }^{12}$. Different factors affect the functional properties of polymeric mucoadhesive drug delivery systems including polymer cross-link density with low crosslinked polymers exhibiting higher flexibility and hydration, with consequent high degree of swelling ${ }^{13}$.

Carrageenan (CAR) is a sulphated natural polymer produced from red seaweed commonly employed in the food industry as a thickening agent ${ }^{14,15}$. Different grades are classified based on the number of sulphate groups present. kappa ( $\kappa)$ CAR (one sulphate group), produces a thermo reversible sol-gel in aqueous solution which undergoes dispersion following random-coil formation in the sol stage. At low temperature, galactose sequences within the carrageenan chains twist in a double helix fashion. The sweet taste of galactose may help to mask the bitter taste of some drugs thus avoiding the need for flavouring and sweetening agents ${ }^{16}$. Several sites for hydrogen bonding impart bioadhesive characteristics though this could be improved by ionic bond formation between the negatively charged 
sulphate group and the positively charged mucin present on the buccal mucosa ${ }^{17}$. Poloxamer 407 (P407) [ $\left.\mathrm{HO}\left(\mathrm{C}_{2} \mathrm{H}_{4} \mathrm{O}\right)_{101}\left(\mathrm{C}_{3} \mathrm{H}_{6} \mathrm{O}\right)_{56}\left(\mathrm{C}_{2} \mathrm{H}_{4} \mathrm{O}\right)_{101} \mathrm{H}\right]$ is a block co-polymer containing polyethylene glycol and propylene oxide ${ }^{18}$. It is a non-ionic surfactant with the ability to increase the solubility of drugs (e.g. ibuprofen) with high $\log \mathrm{P}^{19}$. Previous studies have shown that it exhibits mucosal permeation enhancing properties ${ }^{20}$.

Here, we report on the development and characterisation of polymeric mucoadhesive lyophilized wafers from CAR 911, P407 and polyethylene glycol 600 (PEG 600) for potential buccal mucosa delivery.

\section{Methods}

\section{Materials}

Gelcarin [ $\kappa-$ carrageenan 911 (CAR 911) batch number: 50102070] was a gift from BASF and obtained from the UK distributor Honeywill \& Stein LTD (Surrey, UK). Poloxamer 407 (P407) (batch number: 038k0071) and polyethylene glycol 600 (PEG 600) (batch number: 0001409391) were all purchased from Sigma-Aldrich (Gillingham, UK).

\section{Gel preparation}

Wafers were prepared from aqueous gels containing 1.5-2.5\% w/w CAR 911, 4\% w/w P407 and 0-5.5\% w/w PEG 600. The gel was prepared by dissolving P407 in cold water $\left(<15^{\circ} \mathrm{C}\right)$, left for two hours, CAR 911 added and kept overnight at room temperature to ensure complete hydration. The mixture was heated and continuously stirred using an overhead stirrer at $40-50^{\circ} \mathrm{C}$. PEG 600 (plasticizer) was added to the gel maintained at 40 -

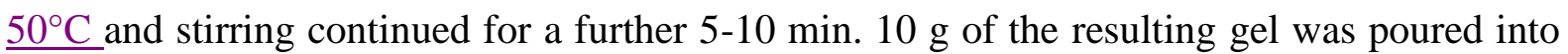
six well plates (diameter $35.4 \mathrm{~mm}$ ). 


\section{Thermal annealing (DSC studies)}

DSC analysis of the gels was conducted with a Q2000 instrument (TA Instruments, Crawley, UK) under a dry nitrogen atmosphere. Identification of the gel's eutectic point $\left(\mathrm{T}_{\mathrm{eu}}\right)$ was used to ascertain the maximum temperature to which the gel could be heated during the primary drying phase of the lyophilization process. 3-10 $\mathrm{mg}$ of gel was loaded into T-zero aluminium pans $(75 \mu \mathrm{L})$, hermetically sealed and cooled initially to $-80^{\circ} \mathrm{C}$ to ensure complete freezing of all components and heated from $-80^{\circ} \mathrm{C}$ to $80^{\circ} \mathrm{C}$ at a rate of $10^{\circ} \mathrm{C} / \mathrm{min}$ and the cycle repeated to determine wafer's stability.

\section{Lyophilization process development}

Preliminary experiments were conducted by freezing the gel in liquid nitrogen, continuing freezing at $-55^{\circ} \mathrm{C}$ and 100 mTorr and eventually the primary drying stage on a Heto Power dry LL3000 freeze dryer (Biopharma Process Systems Ltd. Winchester, UK). Different cycles with varying time durations (24 up to 96 hours) were attempted, but the physical characteristics of the wafers did not meet the required criteria flexibility, plasticity and elasticity and thickness. Subsequently, lyophilization cycle development was pursued with an AdVantage freeze dryer (Biopharma Process Systems Ltd. Winchester, UK) in automatic mode. The freezing, primary and secondary drying cycles were programmed with different time periods, temperature and pressure.

Further optimization was undertaken using a lyophilization cycle incorporating an annealing step in two main ways as follows:

a. The gel (previously kept at room temperature, $5^{\circ} \mathrm{C}$ and $-5^{\circ} \mathrm{C}$ for 30 minutes each) was slowly cooled (at a rate of between $0.6^{\circ} \mathrm{C}-1.3^{\circ} \mathrm{C} /$ minute respectively) to $-35^{\circ} \mathrm{C}$, maintained for 30 minutes, increased to $-10^{\circ} \mathrm{C}$ for 2 hours and returned to the initial 
temperature of $-35^{\circ} \mathrm{C}$. Sample was maintained at $-35^{\circ} \mathrm{C}$ for 2 hours and the vacuum pulled to initiate primary drying to remove the ice crystals by sublimation. This involved increasing the temperature to $-10^{\circ} \mathrm{C}$ for 3 hours, $-5^{\circ} \mathrm{C}$ for 2 hours and finally $0^{\circ} \mathrm{C}$ for 2 hours. The secondary drying stage involved heating from 0 to $5^{\circ} \mathrm{C}$, maintained for 1 hour and consequently heated to $25^{\circ} \mathrm{C}$.

b. Based on the eutectic point $\left(12.8^{\circ} \mathrm{C}\right)$ determined from DSC analysis, the gels were cooled gradually from room temperature to $5^{\circ} \mathrm{C}$, cooled to $0^{\circ} \mathrm{C}$ and maintained for 40 minutes, cooled finally to $-55^{\circ} \mathrm{C}$ and maintained for 1 hour. Annealing was performed by increasing the temperature to $-35^{\circ} \mathrm{C}$ for 3 hours to allow adequate time for large ice crystal formation. The temperature was returned to $-55^{\circ} \mathrm{C}$ and maintained for 2 hours before primary drying was initiated.

During ice crystal sublimation in the primary drying phase, the temperature was increased from $-55^{\circ} \mathrm{C}$ to $-10^{\circ} \mathrm{C}$ and eventually to $0^{\circ} \mathrm{C}$ (lower than the eutectic point) to prevent melt back and preserve the stability of all the components. The whole primary drying procedure was 8 hours at $-10^{\circ} \mathrm{C}$ followed by 6 hours at $0^{\circ} \mathrm{C}$. To increase the stability of wafers at room temperature, secondary drying was performed to further reduce residual water content.

\section{Mechanical characterisation}

Texture analysis was employed to determine the mechanical properties of the wafers by measuring resistance to compression profiles. Two sets of experiments were performed to determine the optimum amounts of each starting material used to formulate the wafers. The preliminary evaluation involved wafers containing different amounts of $\kappa$-CAR 911 with or without PEG 600 to determine their effect on mechanical strength. The second evaluation 
involved the effect of the annealing process on textural (mechanical) characteristics of the wafers. The instrument employed was a Texture analyser HD-plus (Stable Microsystems, Godalming, UK) with Exponent software to plot and display the data. Before compression measurements, the thickness of the wafer was measured by micro screw-meter in five different areas of each sample (four edges and one in the middle) and the average thickness (3.5-3.7 $\mathrm{mm}$ ) entered into the Exponent software. A $6 \mathrm{~mm}$ diameter cylindrical stainless steel probe was used to compress the wafers using the following settings: pre-test speed $(0.1$ $\mathrm{mm} / \mathrm{sec})$; test speed $(0.1 \mathrm{~mm} / \mathrm{sec})$; post-test speed $(1 \mathrm{~mm} / \mathrm{sec})$; depth of compression $(2 \mathrm{~mm})$, trigger force $(0.01 \mathrm{~N})$; hold time $(2 \mathrm{sec})$; mode (return to start). The area under the curve of the force vs distance profiles was calculated.

\section{Thermal analysis (TA)}

(i) Thermogravimetric analysis (TGA)

TGA (TA Instruments, Crawley, UK) was used to determine the residual water in the wafers and the effect of PEG 600 concentration on the water content of the wafers. 3-10 mg was placed in aluminium pans $(100 \mu \mathrm{L})$, heated from $25^{\circ} \mathrm{C}$ to $150^{\circ} \mathrm{C}$ at a rate of $10^{\circ} \mathrm{C} / \mathrm{min}$ and weight loss measured using a high resolution TGA 2950 instrument-(TA Instruments, Crawley, UK).

(ii) Differential scanning calorimetry (DSC)

This was performed to investigate the stability of the starting materials during the lyophilization process. 3-10 mg of wafer was loaded into T zero aluminium pans $(75 \mu \mathrm{L})$, hermetically sealed, cooled to $-80^{\circ} \mathrm{C}$ and heated from $-80^{\circ} \mathrm{C}$ to $180^{\circ} \mathrm{C}$ at a rate of $10^{\circ} \mathrm{C} / \mathrm{min}$. Samples were cooled again to $-80^{\circ} \mathrm{C}$, maintained for 5 minutes before heating again to $180^{\circ} \mathrm{C}$ and maintained for 3 minutes to allow complete melting. This process was repeated to 
investigate the behaviour (stability) of CAR 911, P407 and PEG 600 during the DSC heating cycle and also after storage for six months.

\section{X-Ray powder diffraction (XRPD)}

XRPD was used to investigate the physical form (crystalline or amorphous) of the individual components present in the wafer and to determine any interactions between the initial materials which may lead to formation of new crystalline entities. A D8 Advance XRPD diffractometer (Bruker, Coventry, UK) equipped with a Lyn X-Iris detector and 6.5 $\mathrm{mm}$ slit size was employed to obtain results in reflection and transmission modes. The instrument was set at $40 \mathrm{kV}$ and $40 \mathrm{~mA}$ with primary solar slit of $4^{\circ}$ and secondary solar slit of $2.5 \mathrm{~mm}$ with scattered slit of $0.6 \mathrm{~mm}$. Samples were scanned at a speed of $0.02^{\circ} 2$-theta step size every 0.1 seconds. Wafers were stored in desiccators over silica for six months and re-analysed to determine their stability as in the DSC analysis.

\section{Scanning electron microscopy (SEM)}

SEM was used to evaluate the topographic characteristics and morphology of the wafers. The analyses were carried out using a Jeol Instrument (Japan) with back scattered electrons and artificial shadowing ability with uncoated samples at low vacuum $(<20 \mathrm{~Pa})$ and an accelerating voltage of $20 \mathrm{kV}$.

\section{Hydration and swelling studies}

These were conducted to investigate the maximum time to completely hydrate and their maximum swelling capacity in two media: $0.9 \%$ sodium chloride solution, to mimic biological fluids and phosphate buffer $(\mathrm{pH}=6.2)$ to mimic salivary $\mathrm{pH}$. The buffer was prepared from $\mathrm{KH}_{2} \mathrm{PO}_{4}$ and $\mathrm{NaOH}(0.1 \mathrm{M})$. Samples were cut to $3 \times 3$ pieces, weighed and placed in the liquid media $(42 \mathrm{~mL})$. Weight changes were measured every 20 minutes for a 
maximum of 140 minutes till constant weight and weight change versus time plotted. Percentage swelling (\% weight change) was determined using equation 1 where $\mathrm{W}_{0}$ and $\mathrm{W}_{\mathrm{t}}$ are the weights of the wafers initially and after swelling at time $t$ respectively. Each data point represents the mean $( \pm$ s.d), of three replicates.

$\%$ Swelling $=\frac{\mathrm{W}_{0}-\mathrm{W}_{\mathrm{t}}}{\mathrm{W}_{0}} \times 100$

Eqn 1

\section{In vitro mucoadhesivity studies}

These were conducted using a $75 \mathrm{~mm}$ diameter probe attached to a Texture AnalyserHD Plus instrument. The wafer was cut to $3 \times 3 \mathrm{~cm}$ size and attached to the surface of the probe using double sided adhesive tape. A Petri dish containing agar gel equilibrated with $200 \mu \mathrm{L}$ of buffer solution $(\mathrm{pH}=6.2)$ to simulate $\mathrm{pH}$ conditions in the buccal environment was employed as the mucosal substrate. The sample was then placed on the agar surface, contact maintained for one minute to allow hydration and complete adherence to the surface. The Texture Analyser was programmed to work in tension mode to detach the wafer from the agar surface using the following settings: target mode (distance); pre-test speed $(0.5 \mathrm{~mm} / \mathrm{sec})$; test speed $(0.5 \mathrm{~mm} / \mathrm{sec})$; trigger type (auto); trigger force $(1 \mathrm{~N})$. The maximum force applied to separate the sample from the agar substrate was determined.

\section{Results and discussion}

\section{Lyophilization cycle development and wafer characteristics}

Establishing a suitable thermal profile for the gel was critical for developing an optimized lyophilization cycle. Two critical parameters needed to be determined; eutectic $\left(\mathrm{T}_{\text {eu }}\right)$ and collapse $\left(\mathrm{T}_{\mathrm{c}}\right)$ temperatures. $\mathrm{T}_{\text {eu }}$ defines the crystalline systems detectable by DSC and exceeding it during primary drying causes the compound to melt. Generally, $\mathrm{T}_{\mathrm{eu}}$ 
determines maximum temperature that the formulated product could be heated during primary drying without the loss of structure ${ }^{21}$. Each formulation has a definitive collapse temperature $\left(\mathrm{T}_{\mathrm{c}}\right)$ beyond which the formulation might be unusable (Figure 1a). Maintaining the temperature below the critical limits during freezing or primary drying is essential for maintaining an optimum sample during the lyophilization process ${ }^{22}$.

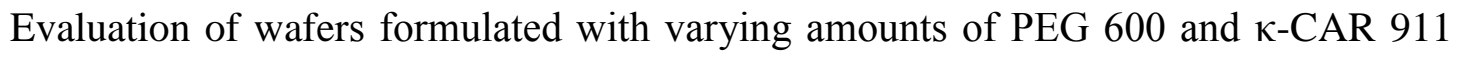
with or without annealing, was conducted according to the following criteria;

i. $\quad$ Flexibility: wafers must be soft and easy to apply onto the mucosal tissues.

ii. Plasticity and elasticity: the wafer should not be fragile and brittle as that affects physical and mechanical stability during handling as well as potential contact irritation during application.

iii. Thickness: an ideal wafer must have optimum thickness (less than $2 \mathrm{~mm}$ ) as thickness greater than $2 \mathrm{~mm}$ could be inconvenient when applied to the buccal mucosal area. Thick wafers also present the possibility of being dislodged by tongue and teeth movement. Thickness also affects the rate of hydration and the diffusion distance through the resulting swollen gel with significant effects on drug release profiles ${ }^{14}$.

Wafers produced with a non-annealing lyophilization cycle showed unacceptable visual and physical characteristics due to excessive brittleness. Further, the presence of ice crystals within the wafer matrix (Figure 1a) confirmed the necessity of incorporating an optimised annealing step which increases porosity by increasing the size of ice crystals. Observation of wafers produced with the first freeze-annealing cycle confirmed that the process was not suitable due to product collapse (Figure 1b) from melt back and/or incomplete ice removal during the primary drying phase. This is because the product temperature must be maintained below the $T_{e u}$ to retain interstitial space in the solid phase and make it capable of supporting 
its own weight after ice removal and preserve the wafer's structure. Therefore, a different lyophilization cycle was developed which was based on the requirement of lower freezing and annealing temperature with a consequent increase in cycle time. When gels were cooled down to $-55^{\circ} \mathrm{C}$ and annealed by increasing the temperature to $-35^{\circ} \mathrm{C}$ and cooled back to $55^{\circ} \mathrm{C}$, wafers were produced with an acceptable texture and physical properties i.e. flexibility, porous structure, without any trace of ice crystals (Figure 1c).

Pressure conditions were critical during the whole lyophilization process and were maintained at 200 mTorr during the freezing stage and was reduced to 50 mTorr during the drying stages as the pressure of the chamber should be higher than the pressure in the frozen gel. This results in sublimation of the ice crystals from the surface of the frozen gel. The primary drying process was conducted by gradual temperature elevation from $-10^{\circ} \mathrm{C}$ to $0^{\circ} \mathrm{C}$ which is at least $10^{\circ} \mathrm{C}$ less than the $\mathrm{T}_{\text {eu }}$ while the appropriate pressure condition was applied which resulted in significant reduction in water content. During secondary drying, desorption occurred and the last traces of water vapour were removed. This stage of freeze drying provided heat to maintain the wafers' at ambient temperature and produce formulations with desirable texture and stability during storage. Overall, the optimised lyophilization cycle incorporating the annealing step totalled 42 hours and produced porous wafers (Figure 1c) with a balance between flexibility and residual water content.

\section{Mechanical properties}

Figure 2a shows that wafers formulated without PEG 600 were very rigid with very high 'work of compression' values, suggesting highly strong and brittle characteristics. Nonannealed wafers were difficult to compress upon application of force (Figure 2b) due to their non-porous texture. Therefore, addition of PEG 600 and incorporating an annealing step into 
the lyophilization cycle significantly impacted upon the mechanical properties of the wafers. The results showed that wafers prepared from gels containing $2 \%$ w/w CAR $911,4 \%$ w/w P407 and 4.4\% w/w PEG 600 exhibited optimum mechanical properties (Figure 2a) while the other wafers were highly brittle and rigid. Although wafers prepared from gels containing 1.5\% w/w CAR 911, 4\% w/w P407 and 3.3\% w/w PEG 600 showed similar compression profiles, other characteristics showed differences with the formulation of choice (see below).

\section{Thermal analysis}

(i) Thermogravimetric analysis (TGA)

The residual water within the wafers produced either by annealing or non-annealing process is summarised in Table 1. Water content following lyophilization is typically expected between $0.5 \%$ and $3 \%{ }^{23}$. The results show that this was considerably higher in nonannealed wafers as well as in formulations containing higher concentrations of CAR 911 and PEG 600, which can be expected owing to the hydrophilic nature of both polymers. Wafers prepared from gels containing 2\% w/w CAR 911, 4.4-0 \% w/w P407 and 4.4\% w/w PEG 600 retained the lowest amount of water and deemed the optimum formulation to assure stability over a longer period. High residual water may act as seeds to initiate and accelerate crystallization which may cause polymorphism within the system resulting in product instability. Water is also an effective plasticizer which significantly depresses the $T_{g}$ of the active compound and excipients by increasing molecular mobility which results in product instability including possible melt back during the primary drying stage ${ }^{24}$. Therefore, annealing is a desirable process to develop wafers with lower residual water content and potentially a more stable product.

(ii) Differential scanning calorimetry (DSC) 
The DSC results (Figure 3) for the selected optimised wafer showed three sharp peaks $\left(-0.19^{\circ} \mathrm{C}, 39.9^{\circ} \mathrm{C}\right.$ and $\left.31.48^{\circ} \mathrm{C}\right)$ corresponding to PEG $600, \mathrm{P} 407$ and the mixture of these two polymers $^{25}$ respectively. The observed peak for PEG 600 can be attributed to the melting of the frozen material obtained following cooling to $-80^{\circ} \mathrm{C}$ during the initial stage of the DSC analysis. We have previously showed similar observations of a third entity corresponding to the mixture of PEG 600 and P407 due to interactions between the two polymers ${ }^{26}$. In addition, DSC profiles for wafers after six months storage showed similar results which confirmed the stability of the polymeric matrix within this time period.

\section{X-ray powder diffraction (XRPD)}

Figure 4a represents the XRPD diffractograms of the starting materials (CAR 911, P407 and PEG 600) indicating amorphous structures for CAR 911 and PEG 600 and crystalline structure for P407. Figure $4 \mathrm{~b}$ shows the diffractogram for wafer freshly prepared from aqueous gel containing all three components (2\% w/w CAR 911, 4\% w/w P407 and 4.4\% w/w PEG 600) with the crystalline molecules attributed to P407. The chemical structure of P407 comprises 79\% PEG and 21\% PPG (polypropylene oxide) and the peaks observed were due to PEG crystals from P407 based on its the XRPD library data base. Similar results were observed after six months of storage, confirming no significant instability during the storage period. The other polymers (CAR 911 and PEG 600) were largely present in amorphous form and expected to help improve swelling capacity due to ease of hydration through chain relaxation.

\section{Scanning electron microscopy (SEM)}

Figure 5 (a-f) shows that increasing the concentration of CAR 911 resulted in a decrease in the pore size. Larger pores can potentially be occupied by higher amounts of 
active compounds and also allow faster water ingress which consequently affect the release of active compound after drug administration. In addition, this is also dependent on the amounts of CAR 911 present as wafers with lower amounts of the polymer are expected to swell more slowly and to a lesser extent overall. This however, requires further investigations as drug release was not the focus of this report.

\section{Hydration and swelling studies}

Since the secretion of saliva is $0.3(\mathrm{ml} / \mathrm{min})^{26}$ and the volume of the medium was 42 $(\mathrm{mL})$ the wafers were immersed for 140 minutes. The results showed that the maximum swelling in acidic medium ( $\mathrm{pH}=5.6$ for saline solution) (Figure 6a) occurred over a longer time period (120 minutes) and the overall swelling capacity was lower in comparison to wafers immersed in buffer media (Figure 6b). Wafers placed in buffer solution at $\mathrm{pH}=6.2$ showed a maximum swelling capacity in 60 minutes that was $300 \%$ higher than in saline solution. This proved the effect of buffer $\mathrm{pH}$ on swelling capacity.

Following the placement of polymeric wafer matrix in a moist environment (such as the buccal mucosa), the swelling process begins by the ingress of water (body fluids). In the early stages, water penetrates into the wafer as a consequence of a concentration gradient resulting in enhanced mobility of the polymer chains followed by an increase in macromolecular mobility at a specific polymer-water concentration. This process is termed polymer chain relaxation (hydration). Consequently, the water content and mesh size of the polymer network within the formulation increase. The relaxation stage is facilitated when the polymer's $T_{g}$ is below the temperature of the swelling media ${ }^{15}$.

Figure 6a also showed that unplasticized wafers formulated with lower amounts of CAR 911 showed the fastest rate of swelling and this trend was steady during the entire measurement period. The effect of CAR 911 on hydration profiles was more dominant 
overall. The above observations can be attributed to differences observed in the microstructure from the SEM images. According to the SEM results (Figure 5a-5f), increasing CAR 911 concentration resulted in wafers with smaller pores and therefore, less capacity for water ingress and consequently hydrated to a lesser extent. The highest swelling capacity of approximately $1000 \%$ and $1300 \%$ in saline and buffer solutions respectively was exhibited by wafers comprising 1.5\% w/w CAR 911 and 4\% w/w P407. As noted earlier, addition of PEG 600, however, decreased the percentage swelling considerably as shown by wafers comprising CAR 911 at 2.5\% w/w and 5.5\% PEG 600 (Figure 6a).

\section{In vitro mucoadhesion studies}

These were performed to predict the stickiness and ability of the wafers to adhere to the buccal mucosal surface. The stickiness factor is defined as the maximum force $(\mathrm{N})$ required for detaching the wafer from the surface of the agar, while cohesiveness is defined as the distance (mm) the wafer travels to detach from the agar surface. Work of adhesion, (energy required to overcome attractive forces between the wafer and agar surface) was measured by calculating the area under the force-distance curves. All of these factors correlate the mucoadhesion characteristics with the strength of the bonds formed between the polymeric matrix and agar during the contact period ${ }^{27}$. The work of adhesivity and stickiness factor were also affected by PEG 600 (Figure 7). Maximum mucoadhesivity was achieved for the wafers prepared from gels comprising $2 \% \mathrm{w} / \mathrm{w}$ of $\kappa$-CAR $911,4 \% \mathrm{w} / \mathrm{w}$ P407 and $4.4 \%$ PEG 600, the same formulation with optimum mechanical characteristics deemed ideal for effective application to the buccal mucosa area.

Mucoadhesion is also closely related to the swelling index (capacity) since excessive hydration and swelling produce a slippery mucilage, which can easily be dislodged from mucosal surfaces. The mechanism of mucoadhesion involves an initial contact (bond 
formation) phase and subsequent consolidation stage. These two stages of the adhesion process are affected by reduced physico-chemical interaction between the slippery gel and the mucosal substrate ${ }^{28,29}$. According to the chemical structure of mucin found on the buccal

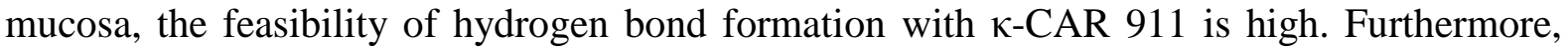
formation of dative covalent bonds between the sulphate group in $\kappa-\mathrm{CAR} 911$ and $\mathrm{NH}_{2}$ groups in mucin is also expected to result in stronger mucoadhesion forces. Ruiz and Ghaly ${ }^{30}$ have also confirmed the ability of CAR tablets to adhere to agar gel surface. Mucoadhesion can also be enhanced through van der Waals forces or entanglement between the wafer matrix and $\operatorname{agar}^{31}$. The $\mathrm{pH}$ at the mucoadhesive interface also affects the adhesion of hydrophilic polymers owing to generation of ionisable groups. Since $\kappa-C A R ~ 911$ is a polyanion and the local $\mathrm{pH}=6.2$ is above the $\mathrm{pKa}$ of 6.1 , it will be slightly ionized and result in enhanced mucoadhesion. Shaikh and co-workers" ${ }^{32}$ stated that "the maximum mucoadhesive strength of polyanions is observed around $\mathrm{pH} \mathrm{4-5,} \mathrm{however,} \mathrm{it} \mathrm{decreases}$ gradually above the $\mathrm{pH}$ of 6". The application of $\kappa$-CAR 911 based buccal dosage form in media with $\mathrm{pH}=6.2$ is not expected to have a negative impact on mucoadhesion force since the $\mathrm{pH}$ is not considerably higher than 6 .

The concentration of PEG 600 directly correlated with the amount of residual water within the polymeric matrix. In the presence of higher amounts of PEG 600, the quantity of water increased (TGA results) resulting in a slight decrease in the mucoadhesive performance $^{33}$. Therefore, PEG 600 concentration in the system should be kept at the lowest optimum level. The overall results also suggest that porosity plays a critical role due to its effect on the initial hydration from water ingress, allowing the formation of hydrogen bonds required for adhesive interactions between the matrix and the mucosal substrate. 


\section{Conclusion}

The development of lyophilised wafers by lyophilization (with thermal annealing) gels containing 2\% w/w CAR 911 and 4\% w/w P407 and $4.4 \%$ w/w PEG 600 with optimum physico-mechanical properties has been achieved. This was confirmed by the hydration, mucoadhesion and TGA studies. The wafers were stable during six months storage and showed optimum swelling and mucoadhesion in conditions simulating those of saliva compared with saline, and have potential for buccal mucosa drug delivery.

\section{References}

[1] Basu A, Wilkinson M, Penugonda K, Simmons B, Betts N. M, Lyons T. J. Lyophilized strawberry powder improves lipid profile and lipid peroxidation in women with metabolic syndrome: baseline and post intervention effects. Nutr J 2009; 8: 43.

[2] Hu W, Wan Z, Hollister S J and Krebsbach P H. Localized viral vector delivery to enhance in situ regenerative. Gene Ther 2007; 14: 891-901.

[3] Lindsay J P, Clark D S, Dordick J S. Combinatorial formulation of biocatalyst preparations for increased activity in organic solvents: Salt activation of penicillin amidase. Biotechnol Bioengvv 2004; 85: 553-560.

[4] Adams G D. Lyophilization of Vaccines. Current Trends Methods Mol Med 2003; 87: 223-243.

[5] Dixi R P, Puthli S P. Oral strip technology: Overview and future potential J Cont Rel 2009; 139: 94-107.

[6] Luppi B, Bigucci F, Abruzzo A, Corace G, Cerchiara T, Zecchi V. Lyophilized chitosan/pectin nasal inserts for antipsychotic drug delivery. Eur J Pharm Biopharm 2010; 75: $381-387$. 
[7] Tsinontides S C, Rajniak P, Pham D, Hunke W A, Placek J, Reynolds S D. Freeze dryingprinciples and practice for successful scale-up to manufacturing. Int J Pharm 2004; 280:1-16.

[8] Schneid S C, Gieseler H. An overview of the annealing process and rational design of thermal treatment steps in freeze-drying. VirTis / FTS Company [Online] 2009; 1-4. Available at: http://www.spscientific.com/LyoTech-Center/Tech-BriefAbstracts/Abstract--Using-Differential-Scanning-Calorimetry-.aspx. Accessed on 12 November 2011.

[9] Barbaree J M, Sanchez M. A guide to freeze-drying in the laboratory. Labonco [Online] 2004; 1-11. Available at: http://www.nbtc.cornell.edu/facilities/freeze_drying_guide.pdf. Accessed on 23 July 2012.

[10] Ayensu I, Mitchell J C, Boateng J S. In vitro characterisation of chitosan based wafers for potential buccal delivery of proteins. Carbo Polym 2012; 89: 935-941.

[11] Pyne A, Surana R, Suryanarayanan R. Crystallization of Mannitol below Tg' during Freeze-Drying in Binary and Ternary Aqueous Systems. Pharm Res 2002; 19: 901-908.

[12] Mathias N R, Hussein M A. Non-invasive Systemic Drug Delivery: Developability considerations for alternate routes of administration. J Pharm Sci 2009; 99: 1-20.

[13] B. Narasimhan and N. A. Peppas, Molecular analysis of drug delivery systems controlled by dissolution of the polymer carrier. J Pharm Sci 1997; 86 297-304.

[14] Shaikh R, Raj Singh T R, Garland J, Woolfson A D, Donnelly R F. Bioallied Sci: Mucoadhesive drug delivery systems. J Pharm 2011; 3: 89-100.

[15] Yuguchi Y, Thuy T, Urakawa H, Kajiwara K. Structural characteristics of carrageenan gels: temperature and concentration dependence. Food Hydrocoll 2002; 16: $515-522$ 
[16] Tari O, Kara S, Pekcan O. Critical exponents of kappa carrageenan in the coil-helix and helix-coil hysteresis loops. J Macromol Sci Part B Phy 2009; 48: 812-822.

[17] Thommes M, Kleinebudde P. Use of kappa-carrageenan as alternative pelletisation aid to microcrystalline cellulose in extrusion/spheronization. II. Influence of drug and filler type. Eur J Pharm Biopharm 2006; 63: 68-75.

[18] Liu Y, Lu WL, Wang H C, Zhang X, Zhang H, Wang X Q, Zhou T Y, Zhang Q J. Controlled delivery of recombinant hirudin based on thermo-sensitive Pluronic (R) F127 hydrogel for subcutaneous administration: in vitro and in vivo characterization. J Contr Rel 2007; 117: 387-395.

[19] Tirnaksiz F, Robinson J R. Rheological, mucoadhesive and release properties of Pluronic F-127 gel and Pluronic F-127/polycarbophil mixed gel systems. Pharmazie 2005; 60: 518-523.

[20] Sohi H, Ahuja A, Jalees Ahmad F, Krishen Khar R. Critical evaluation of permeation enhancers for oral mucosal drug delivery. Drug Dev Ind Pharm 2010; 36: 254-282.

[21] Roy S, Pal K, Anis A, Pramanik K, Prabhakar B. Polymers in Mucoadhesive Drug Delivery System: A Brief Note. Des Monomers Polym 2009; 12: 483-495.

[22] Schewegman J J. Understanding the physical properties of properties of materials in lyophilized products. SP Company [Online] 2009; 1-4. Available at: ww.pharmaceuticalonline.com/article.mvc/Basic-Cycle-Development-Techniques-ForLyophi-0001. Accessed on 28 October 2011.

[23] MC Ginn J. Freeze-dry microscopy improves pharmaceutical efficiency, cost and quality. The McCrone Group [Online] 2009; Available at: http://www.mccronemicroscopes.com/store/images/pdf. Accessed on 15 August 2011.

[24] Biopharma Process Systems (www.biopharma.co.uk). Accessed on 20/06/2011. 
[25] Eliasson L, Birkhed D, Carlen A. Feeling of dry mouth in relation to whole and minor gland saliva secretion rate. Arch Oral Bio 2009; 54: 263-267.

[26] Kianfar F, Chowdhry B Z, Boateng J S, Antonijevic M D. Investigation of the interaction between poloxamer 407 and poly (ethylene glycol) 600. J Pharm Pharmacol 2010; 62: 1341 .

[27] Bansal K, Rawat M K, Jain A, Rajput A, Chaturvedi T P, Singh S. Development of Satranidazole Mucoadhesive Gel for the Treatment of Periodontitis. AAPS PharmSciTech 2009; 10: 716-723.

[28] Smart J D. The basics and underlying mechanisms of mucoadhesion. Adv. Drug Deliv Rev 2000; 57: 1556-1568.

[29] Andrew G P, Laverty T P, Jones D S. Mucoadhesive polymeric platforms for controlled drug delivery. Eur J Pharm Biopharm 2009; 71: 505-518.

[30] Ruiz G, Ghaly E. Mucoadhesive delivery systems using carrageenan and eudragit. Vitae. RLPO 2006; 13:1-2.

[31] Smart J D. Buccal drug delivery. Expert Opin Drug Deliv 2005; 3: 507-17.

[32] Shaikh R, Raj Singh T R, Garland M J, Woolfson D A, Donnelly R F. Bioallied Sci: Mucoadhesive drug delivery systems. J Pharm 2011; 3: 89-100.

[33] Sigurdsson H, Loftsson T, Lehr C. Assessment of mucoadhesion by a resonant mirror biosensor. Int J Pharm 2000; 325: 75-81. 


\section{Figure legends}

Figure 1 Digital images of wafers prepared from gels comprising 2\% w/w CAR $911+4 \%$ w/w P407 + 4.4\% w/w PEG 600 (a) without annealing (b) non-optimised annealing cycle and (c) optimised annealing cycle.

Figure 2 Work of compression (N.mm) profiles for wafers produced from gels comprising (a) 1.5-2.5\% w/w CAR $911+4 \%$ w/w P407 + 0-5.5\% w/w PEG 600 (b) 2\% w/w CAR $911+$ 4\% w/w P407 + 4.4\% w/w PEG 600 produced by annealing or non-annealing cycle.

Figure 3 DSC thermogram of wafer produced from gels containing 2\% w/w CAR $911+4 \%$ w/w P407 + 4.4\% w/w PEG 600 .

Figure 4 XRPD patterns of (a) starting materials (CAR 911, P407 and PEG 600) and (b) wafer produced from gels containing $2 \% \mathrm{w} / \mathrm{w}$ CAR $911+4 \% \mathrm{w} / \mathrm{w} \mathrm{P} 407+4.4 \% \mathrm{w} / \mathrm{w}$ PEG 600.

Figure 5 SEM images showing the surface morphology of the wafers prepared from gels containing (a) $1.5 \%$ w/w CAR $911+4 \%$ w/w P407, (b) $1.5 \%$ w/w CAR $911+4 \%$ w/w P407 + 3.3\% w/w PEG 600 (c) 2\% w/w CAR $911+4 \%$ w/w P407, (d) 2\% w/w CAR $911+4 \%$ P407 + 4.4\% PEG 600, (e) $2.5 \%$ w/w CAR $911+4 \%$ w/w P407 and (f) $2.5 \%$ w/w CAR 911+ $4 \% \mathrm{w} / \mathrm{w} \mathrm{P} 407+5.5 \% \mathrm{w} / \mathrm{w}$ PEG 600 .

Figure 6 Hydration profile showing the $\%$ swelling (mean \pm s.d. $n=3$ ) for the wafer containing various concentrations of CAR 911, 4\% w/w P407 with or without PEG 600 in (a) saline solution and (b) phosphate buffer. 
Figure 7 Mucoadhesion profiles: work of adhesion (WOA), stickiness and cohesiveness of wafers containing different concentrations CAR 911 with or without PEG 600.

Table 1 TGA results showing the $\%$ water content in xerogels containing varying concentrations of the polymers $(n=3)$, mean \pm s.d.

\begin{tabular}{|c|c|c|}
\hline Gel formulation content $(w / w)$ & Annealed & Non-annealed \\
\hline $1.5 \%$ CAR $911+4.0 \%$ P407 & $0.7 \pm 0.0$ & $4.7 \pm 0.6$ \\
\hline $1.5 \%$ CAR $911+4.0 \%$ P $407+3.3 \%$ PEG 600 & $2.2 \pm 1.0$ & $4.3 \pm 0.8$ \\
\hline $1.5 \%$ CAR $911+4.0 \%$ P $407+5.5 \%$ PEG 600 & $2.1 \pm 1.2$ & $5.3 \pm 1.3$ \\
\hline $2.0 \%$ CAR $911+4.0 \%$ P407 & $1.5 \pm 0.5$ & $4.7 \pm 1.2$ \\
\hline $2.0 \%$ CAR $911+4.0 \%$ P $407+4.4 \%$ PEG 600 & $1.2 \pm 0.5$ & $5.3 \pm 0.5$ \\
\hline $2.0 \%$ CAR $911+4.0 \%$ P $407+5.5 \%$ PEG 600 & $2.5 \pm 1.0$ & $5.4 \pm 0.5$ \\
\hline $2.5 \%$ CAR $911+4.0 \%$ P407 & $1.4 \pm 0.0$ & $4.5 \pm 0.3$ \\
\hline $2.5 \%$ CAR $911+4.0 \%$ P $407+5.5 \%$ PEG 600 & $1.8 \pm 0.1$ & $5.8 \pm 0.3$ \\
\hline $2.0 \%$ CAR $911+4.0 \%$ P $407+4.4 \%$ PEG 600 & $\begin{array}{c}1.5 \pm 0.5 \\
\text { (freshly prepared) }\end{array}$ & ----- \\
\hline $2.0 \%$ CAR $911+4.0 \%$ P $407+4.4 \%$ PEG 600 & $\begin{array}{c}1.5 \pm 0.8 \\
\text { (one month storage) }\end{array}$ & \\
\hline
\end{tabular}




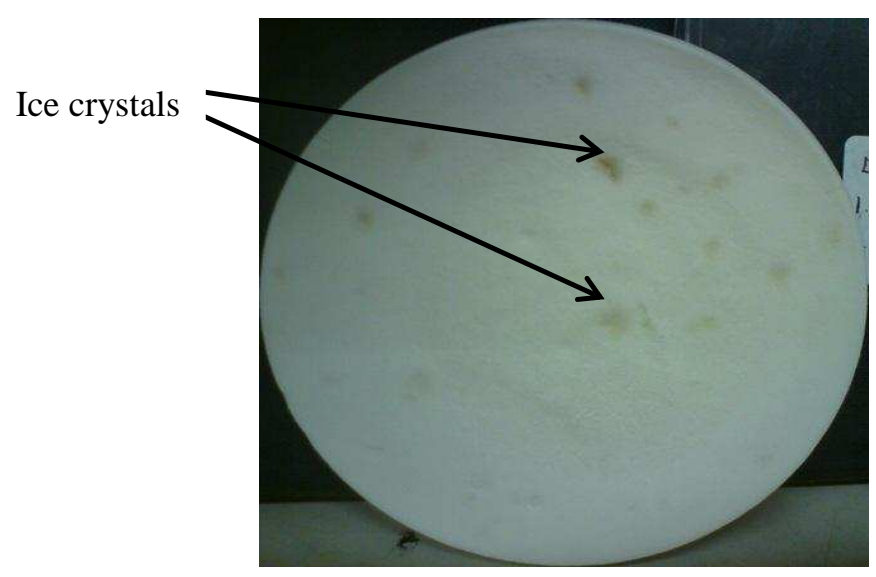

(a)

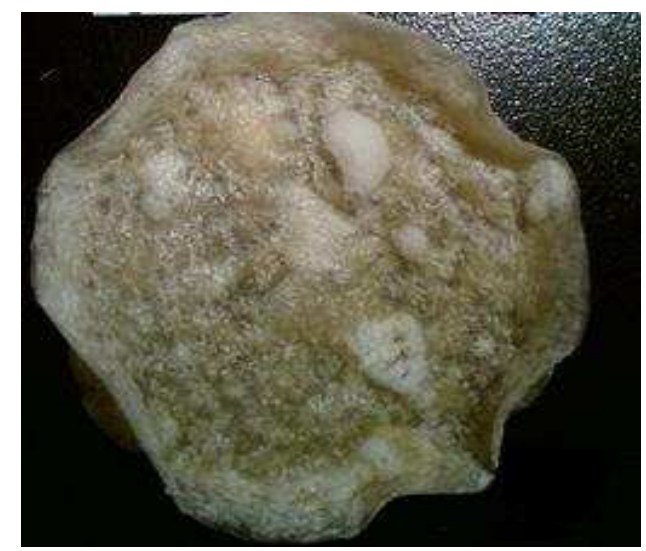

(b)

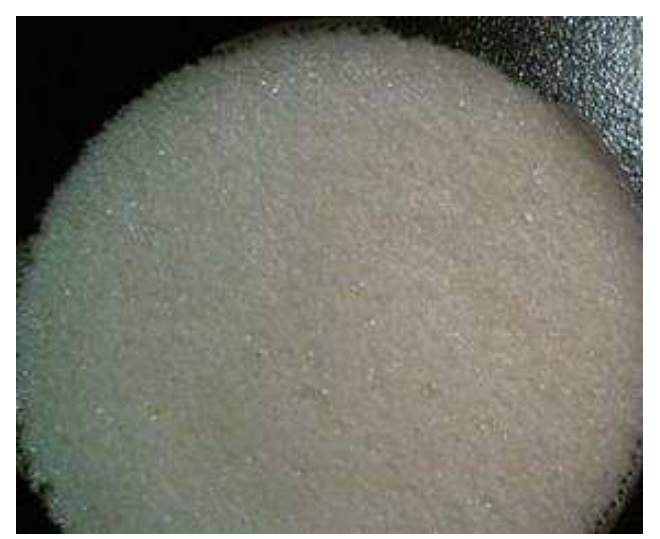

(c)

Figure 1 


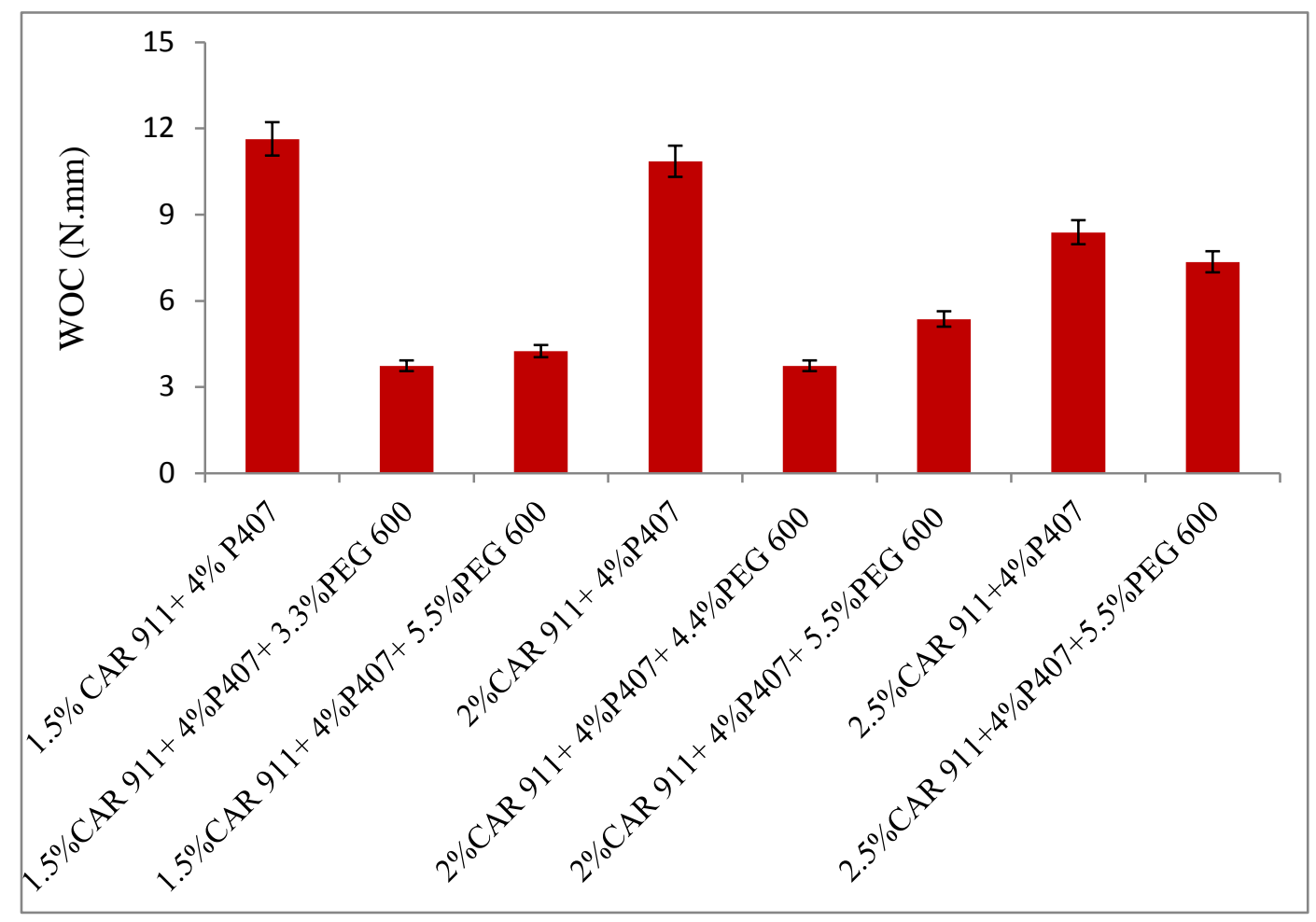

(a)

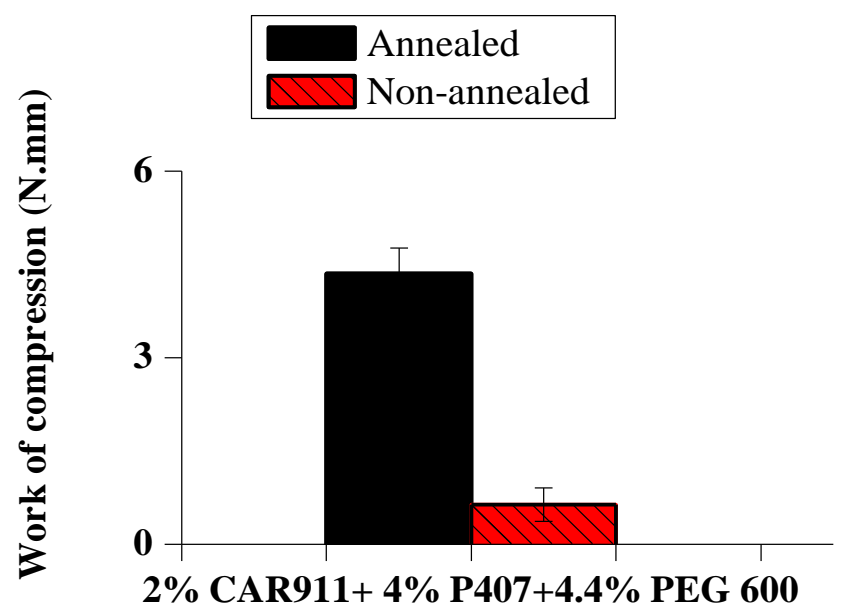

(b)

Figure 2 


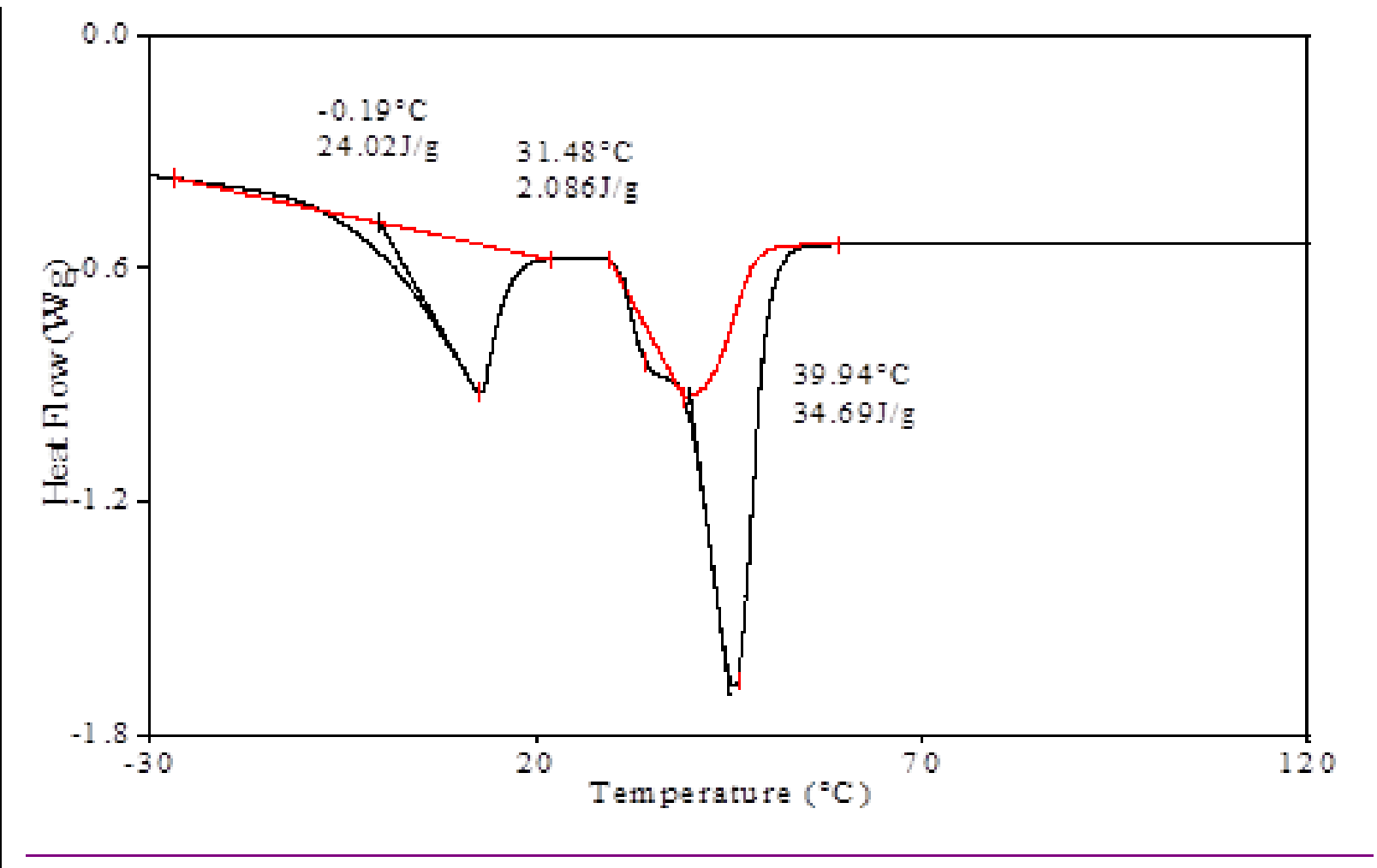

Figure 3 


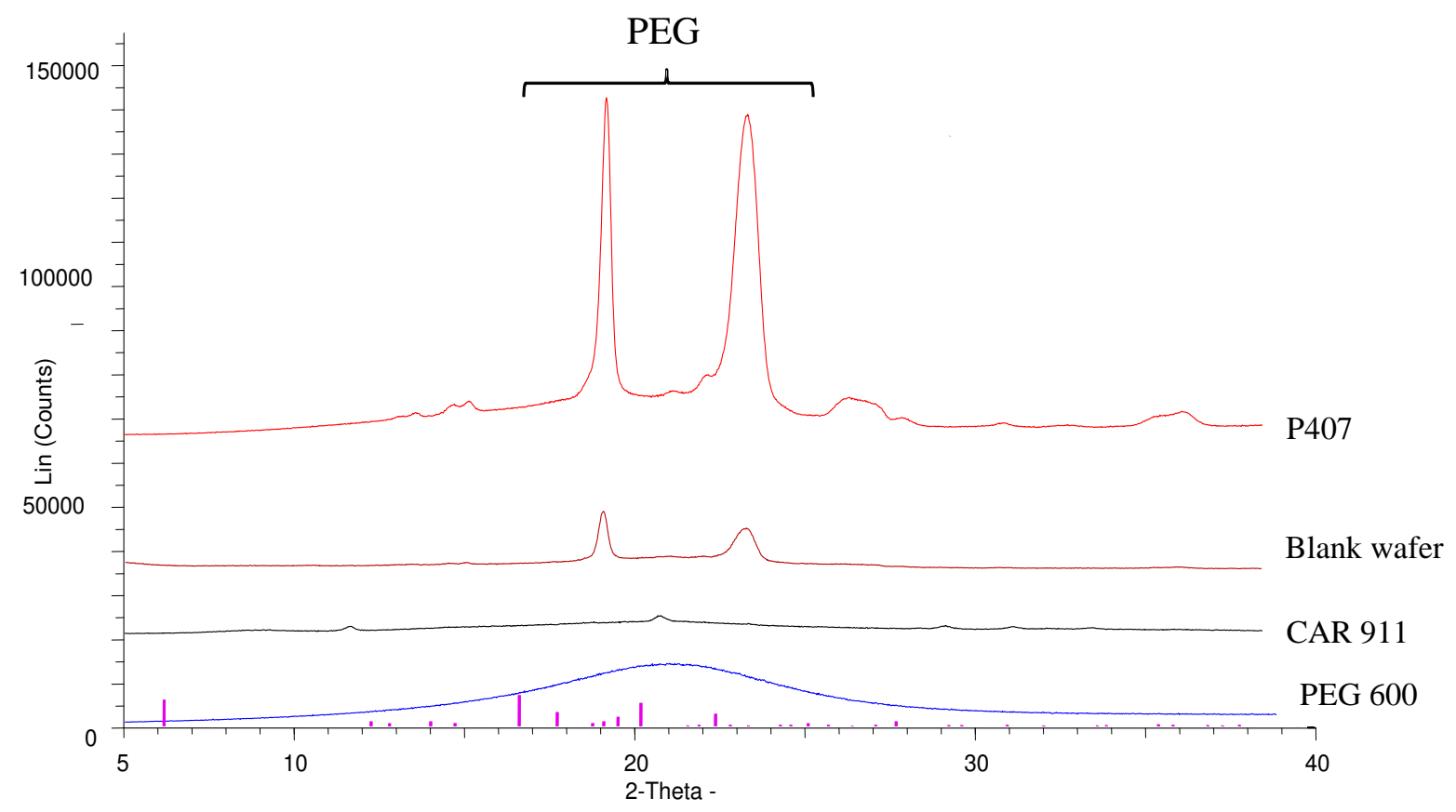

(a) 


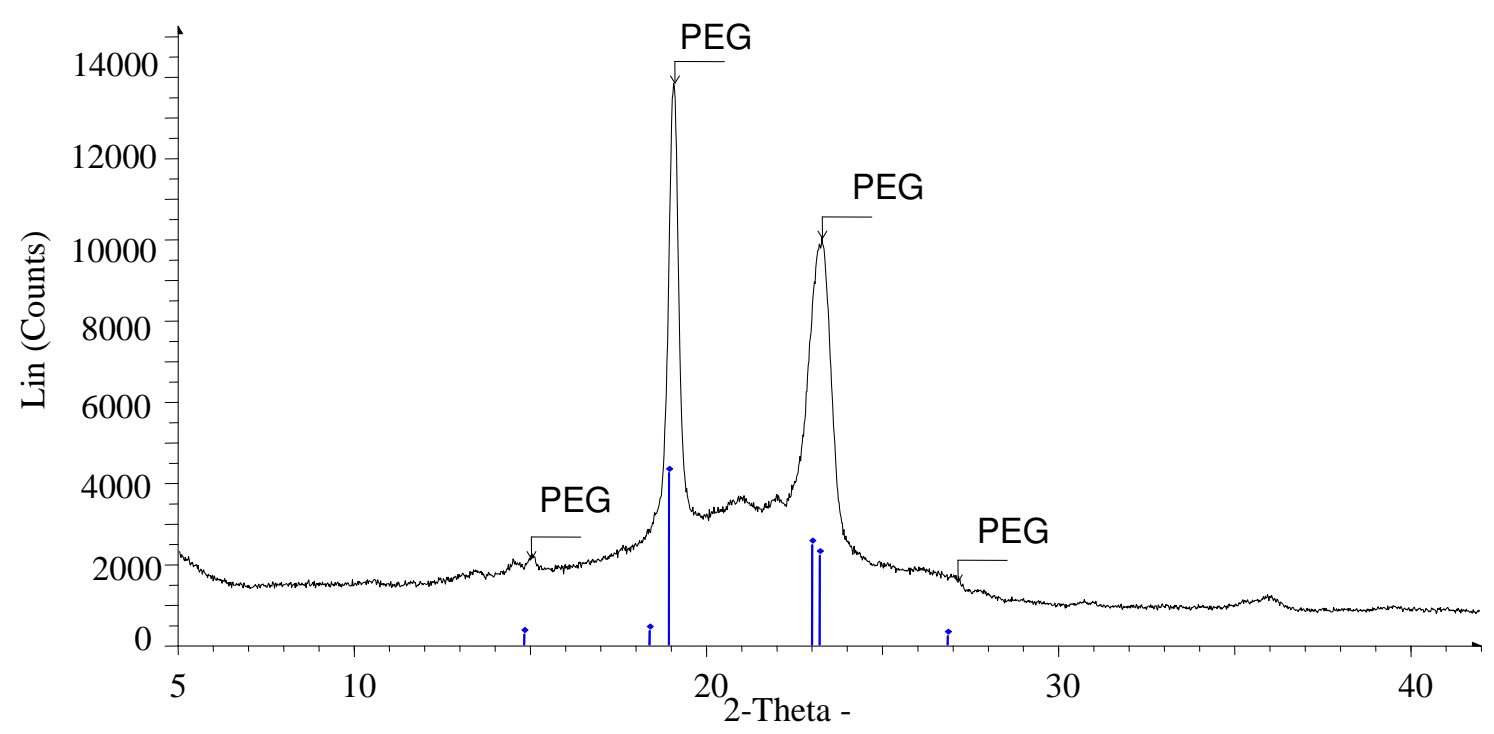

(b)

Figure 4

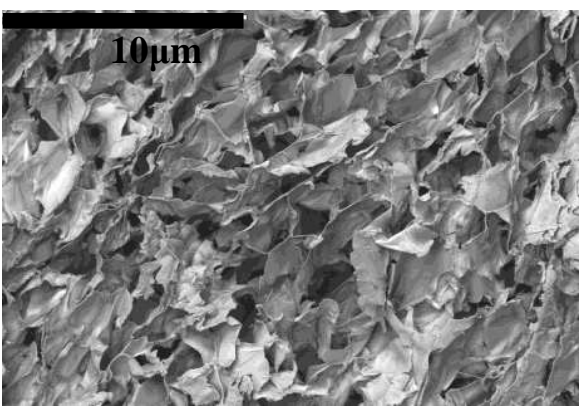

(a)

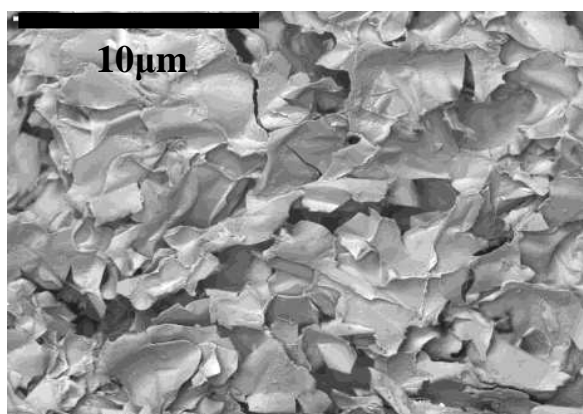

(c)

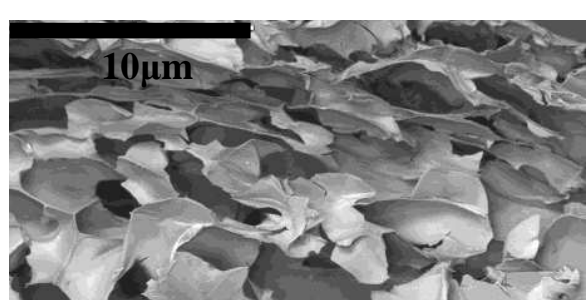

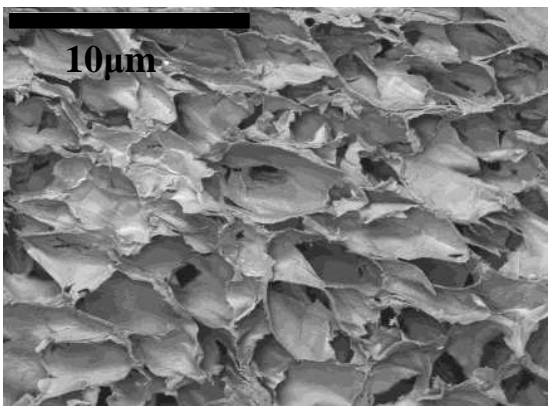

(b)

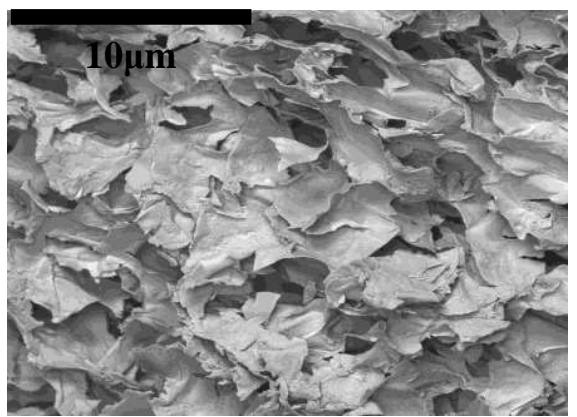

(d)

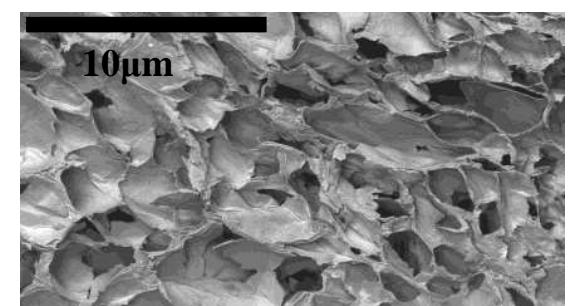


Figure 5 


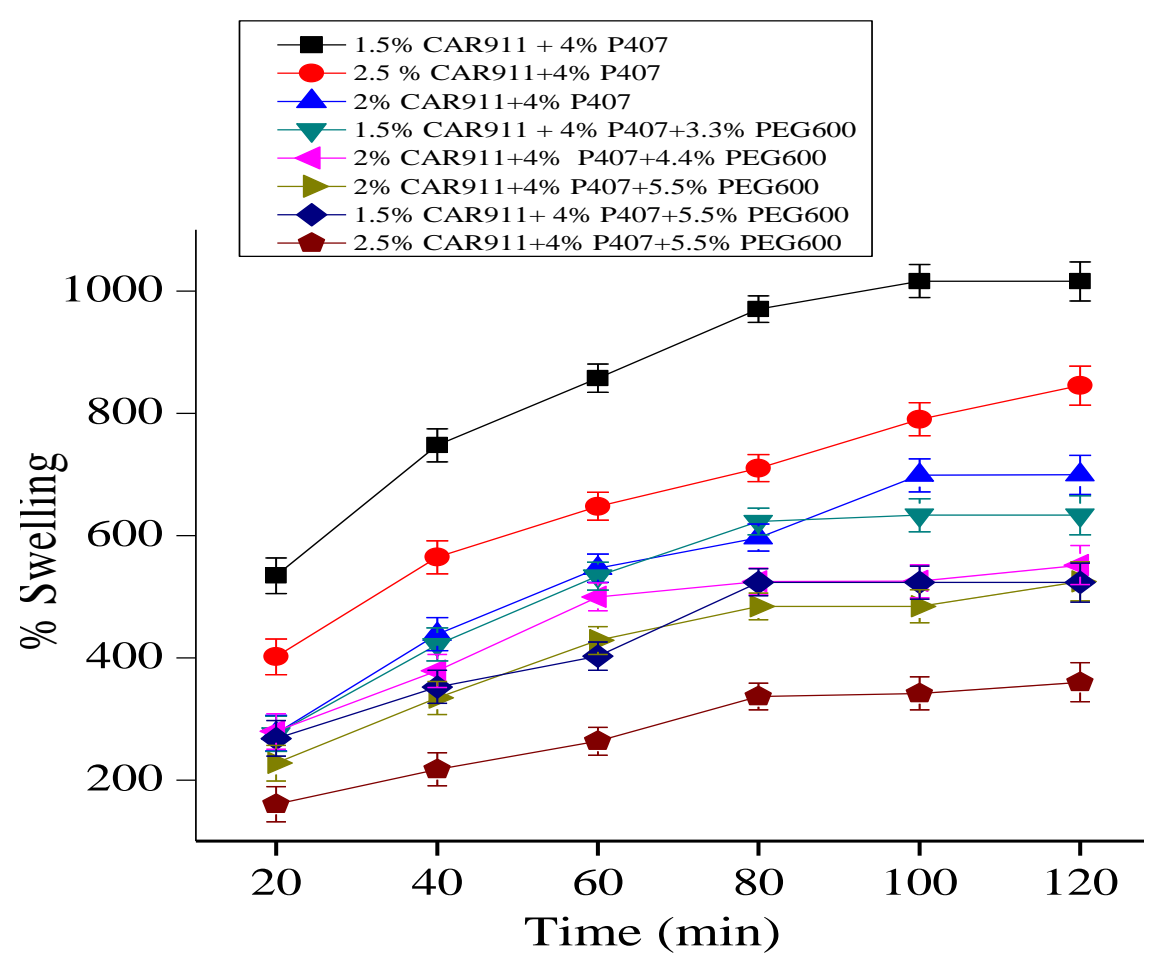

(a)

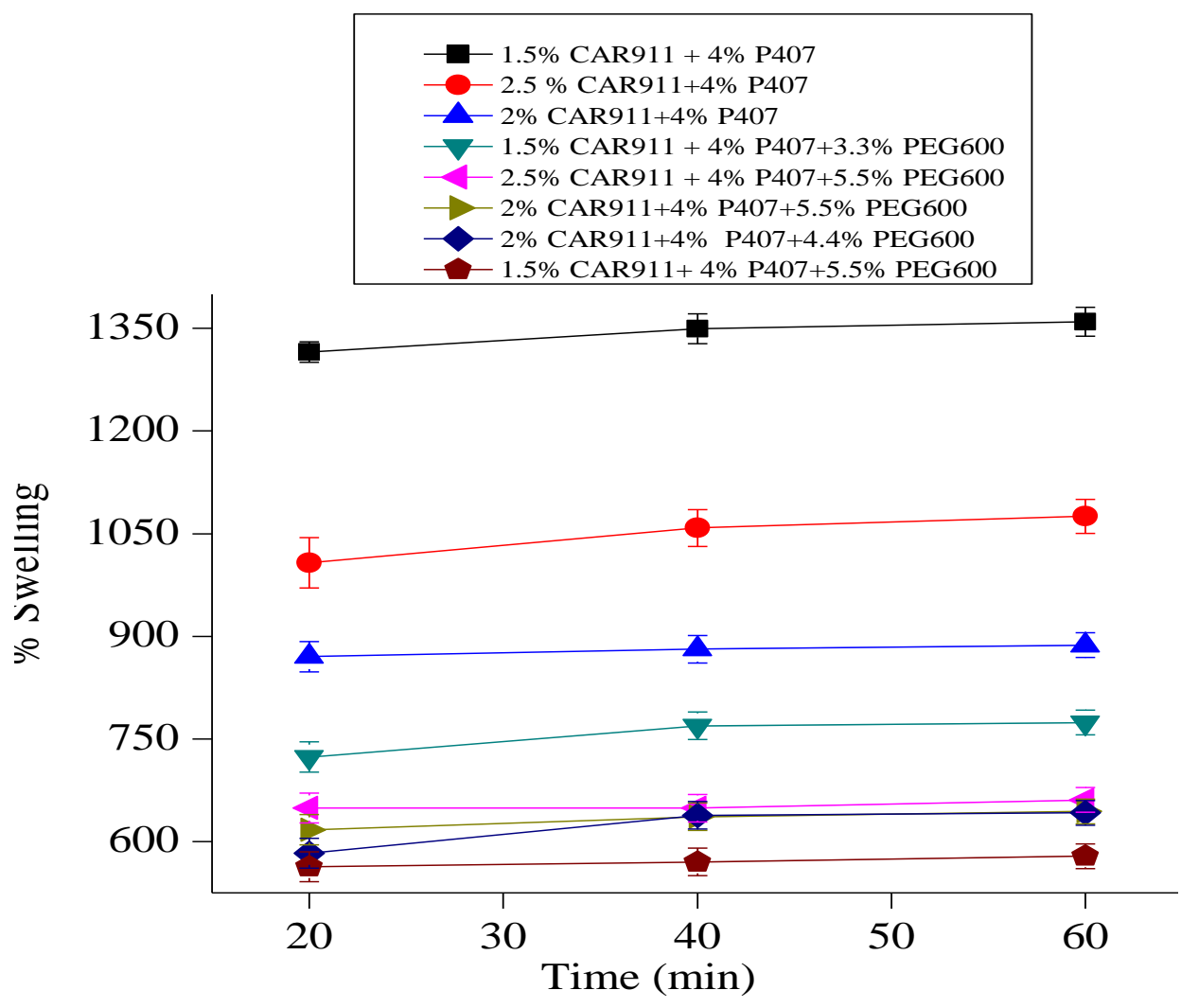

(b)

Figure 6 


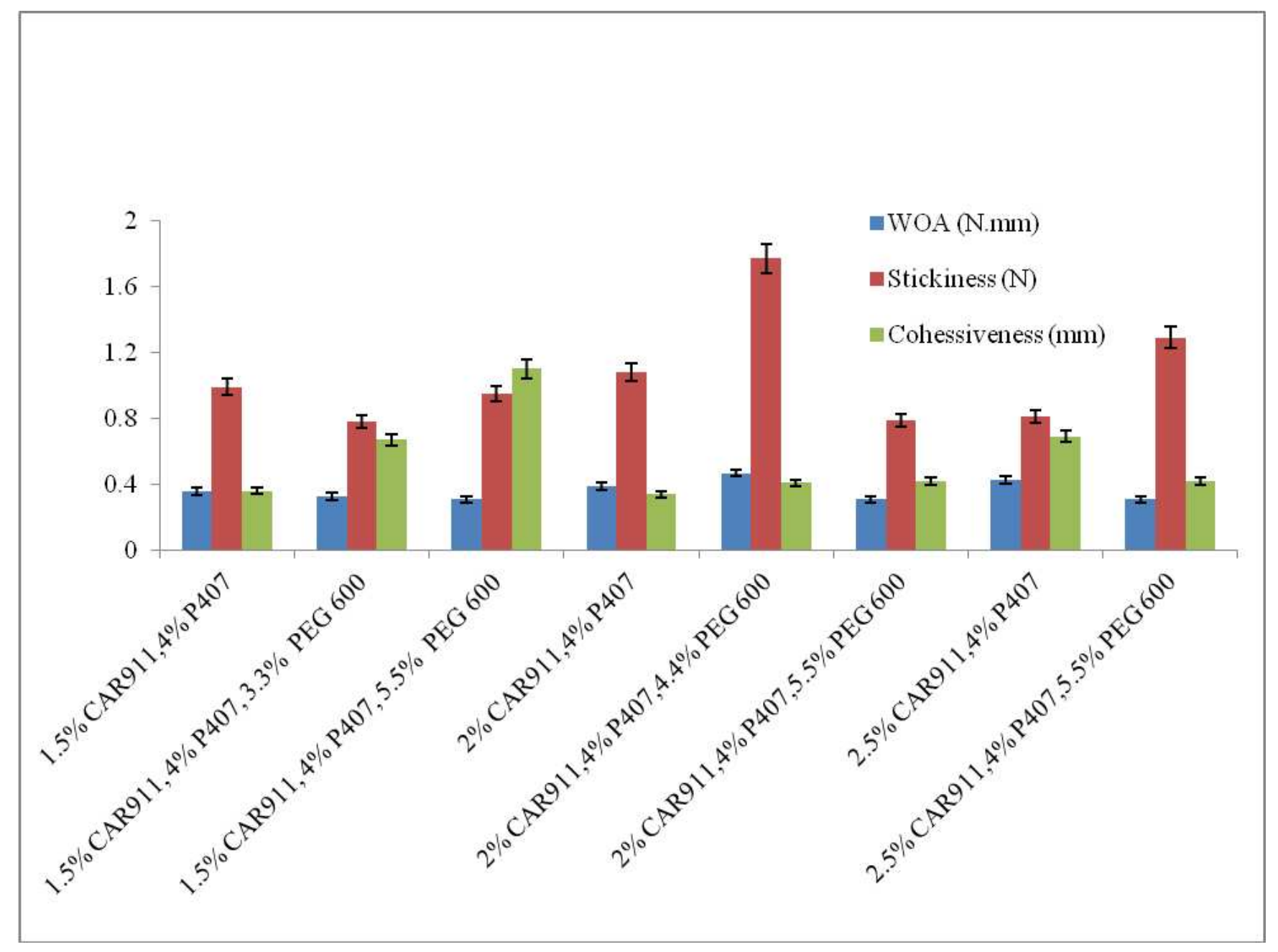

Figure 7 